\title{
Objective measurement of habitual sedentary behaviour and physical activity in pre-school children: Comparison of activPAL with Actigraph monitors
}

\author{
A. Martin ${ }^{1}$, M. McNeill ${ }^{1}$, V. Penpraze ${ }^{1}$, P. Dall ${ }^{2}$, M. Granat ${ }^{2}$, J. Y. Paton ${ }^{1}$ and J. J. Reilly ${ }^{1}$ \\ ${ }^{1}$ College of Medical, Veterinary, and Life Sciences, Yorkhill Hospitals, University of Glasgow, Glasgow G3 8SJ, UK and \\ ${ }^{2}$ School of Health, Glasgow Caledonian University, Cowcaddens Road, Glasgow G4 OBA, UK
}

Sedentary behaviour is associated with increased energy intake and obesity in young children ${ }^{(1)}$. The Actigraph is well established for measurement of both free-living sedentary behaviour and physical activity ${ }^{(2)}$. Estimates of sedentary behaviour and physical activity by the novel activPAL have not yet been published in children but the validity for measurement of both constructs in young children is established ${ }^{(3)}$. The present study aimed at comparing measures of sedentary behaviour and physical activity between the two monitors in a convenience sample of free-living pre-school children.

Twenty-three pre-school children (mean age: 4.5 years) wore the activPAL and the Actigraph accelerometer simultaneously during waking hours for a mean of $5.6 \mathrm{~d}$ and $10 \mathrm{~h} / \mathrm{d}$. Measures of daily mean percentage time sedentary (lying/sitting and standing with no translocation) and physically active were compared between the two monitors at a group and individual level.

Daily mean percentage of time sedentary was 74.6 (SD 6.8) for the Actigraph and 78.9 (SD 4.3) for the activPAL. Daily mean percentage of time physically active (light intensity plus MVPA) was 25.4 (SD 6.8) for the Actigraph and 21.1 (SD 4.3) for the activPAL. Rank order correlations between the two devices were statistically significant $(r=0.676, P<0.001)$. Differences in percentage of time spent sedentary and physically active between the monitors were significant (paired $t$-test, $P<0.001$ ). The Bland-Altman analysis (Fig. 1) showed that for estimates of percentage of the day spent sedentary there was a difference of $-4.3 \%$ (SD 4.8 ) of daily time (limits of agreement -14.0 to $5.4 \%$ ) for the Actigraph relative to the activPAL.

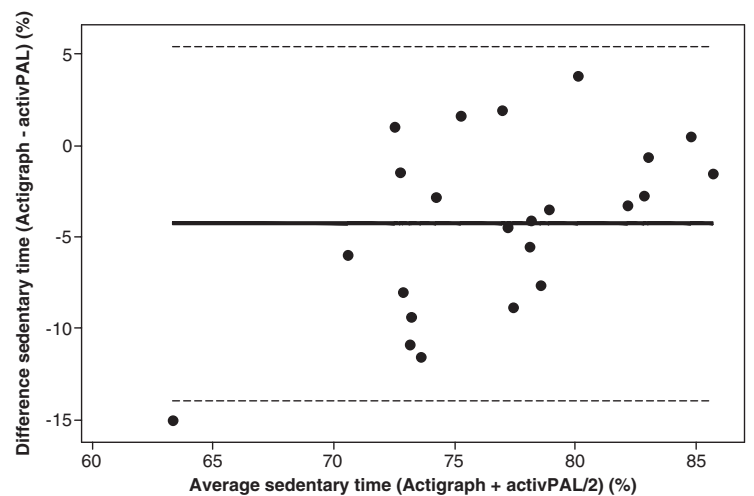

Estimates of percentage of daily time spent sedentary and in total physical activity were broadly similar between the monitors at the group level but differed at the individual level. An apparently small difference may have an important impact for some applications, such as in obesity treatment and assessment of compliance with physical activity and sedentary behaviour guidelines.

1. Australian Department of Health and Aging (2009) Get up and Grow. www.health.gov.au/internet/main/publishing.nsf/Content (accessed 1 December 2010).

2. Reilly JJ, Penpraze V, Hislop JK et al. (2008) Arch Dis Child 93, 614-619.

3. Davies G, Reilly JJ, Granat M et al. (2010) Int J Behav Nutr Phys Act abstract. 